\title{
Liver Metastases from Gynecological Cancers: Time to Resection?
}

\author{
Jose M. Ramia*, Roberto De La Plaza, Jose Quiñones, Pilar Veguillas, \\ Farah Adel, Jorge García-Parreño \\ Unidad de Cirugía Hepatobiliopancreática, Servicio de Cirugía General y Aparato Digestivo, \\ Hospital Universitario de Guadalajara, Guadalajara, Spain

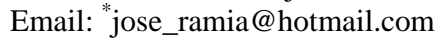

Received November 13, 2011; revised January 27, 2012; accepted February 10, 2012

\begin{abstract}
Aim: To perform an update of liver resection as treatment of liver metastases of gynecological cancers, as well as an update on these metastases. Bibliographical Search: PubMed Search 1990-2011 in English language. Authors reviewed only relevant articles. Results: No more than 20 relevant articles have been published on this topic, including case reports. We performed a classical review of the information published in the literature. Conclusions: Liver Metastases from Gynecological cancers, not peritoneal implants in the liver, usually appear in patients with disseminated disease, so it is uncommon to perform a liver resection. In some patients with limited extrahepatic disease or only liver disease, liver resection is a safe alternative and improves the oncological results. We try to emphasize which patients may benefit from liver resection and the importance of tailoring medical decisions.
\end{abstract}

Keywords: Liver; Metastasis; Gynecology; Ovary; Cancer; Review

\section{Introduction}

Resection of liver metastases from gynecological cancer (LMGC) is a debatable topic. As there are no clear guidelines or randomized trials performed, it was decided to perform this review. A bibliographical search in was carried out in Pubmed using the words: "liver metastasis gynecological cancers" in the period 1990-2011. We found 166 articles, but only 12 were related to the topic. Others searches performed using any kind of cancer (ovary, granulosa cell tumor, cervical...) did not improve the results. Another 12 articles were found on studying the references in the first 12 articles, finally having found 24 articles related to the topic. We also reviewed the most relevant series on liver metastases of non-colorectal, nonneuroendocrine origin that included LMGC. The reviewed data are discussed in this article.

In recent years, technical improvements in liver resection and perioperative management have led to a dramatic decrease in morbidity and mortality associated with these procedures [1-12]. Resection of liver metastases (LM) of colorectal cancer and neuroendocrine tumors is currently the treatment of choice when technically feasible, with 5 years survival ranging from 35\% - 61\% [1-10, 12-14].

However, liver resection in patients with non-colorectal non-neuroendocrine liver metastases, which include pa-

\footnotetext{
"Corresponding author.
}

tients with LMGC, is still a not fully accepted indication in within the medical community despite the results obtained in published series with a median survival at 5 years of 35\% [3,5-9,12,15]. The number of patients undergoing resection of LMGC is very small [3,5-9,15]. It has been estimated that LMGC are less than $1 \%$ of the total LM resected [10]. The low number of LMGC resections is probably due to the lack of defined criteria indicating which patients should be operated on, and the doubt whether a local treatment is useful in a disseminated disease $[1,5,7,10,15,16]$. Moreover, there are no randomized trials comparing chemotherapy alone with LMGC surgical resection [5,17]. The National Comprehensive Cancer Network guidelines for ovarian and uterine cancers do not define clearly what treatment should be performed in LMGC $[18,19]$.

\section{LMGC}

The limited data in the literature on LMGC liver resections are found in two types of articles: LM series on nonneuroendocrine colorectal tumors (NNNC) that include many different LM types of tumors, among them LMGC [3,6-8,15], and a very small number of articles devoted exclusively to LMGC $[5,16]$.

Among the series of NNNC LM (Table 1), the most important is a French multicenter study by Adam et al. including 1452 LM NNNC, 126 of them LMGC. Resec- 
Table 1. LMGC in NNNC LM articles.

\begin{tabular}{|c|c|c|c|c|c|c|}
\hline & LM NNNC & LMGC & LM OC & Other GC & Global 5 year-SV (\%) & Median mo. SV \\
\hline Ercolani, 2005 [15] & 83 & 9 & 8 & 1 Uterus & $33 \%$ & - \\
\hline \multirow{3}{*}{ Adam, 2006 [8] } & \multirow{3}{*}{1452} & \multirow{3}{*}{126} & \multirow{3}{*}{65} & 43 Uterus & $48 \%$ & \\
\hline & & & & \multirow[t]{2}{*}{$18 \mathrm{ND}$} & $50 \%(\mathrm{OC})$ & $98 \mathrm{mo}$ \\
\hline & & & & & 35\% (UC) & $32 \mathrm{mo}$ \\
\hline Earle, 2006 [3] & 77 & 10 & - & & - & - \\
\hline Reddy, 2007 [6] & 82 & 12 & 11 & 1 Endometrium & - & - \\
\hline O’Rourke, 2008 [7] & 102 & 13 & 12 & 1 Endometrium & $38.7 \%$ & 46 mo \\
\hline Kollmar, 2008 [1] & 45 & 16 & & & $30 \%$ & \\
\hline \multirow{2}{*}{ Schmelzle, 2010 [9] } & \multirow{2}{*}{44} & \multirow[b]{2}{*}{7} & \multirow[b]{2}{*}{5} & 1 Endometrium & - & - \\
\hline & & & & & & \\
\hline
\end{tabular}

LM NNNC: liver metastasis non-neuroendocrine non-colorectal; LMGC: liver metastasis gynecological cancer; SV: survival; mo: months; ND: no disposable.

tion of LMGC obtained a 5-year survival of $48 \%$, but differed between 65 patients with LM from OC (ovarian cancer), achieving a 50\% 5-year survival with a median of 98 months, compared to $35 \%$ and 32 months for patients with uterine tumors. No information on the survival of the 18 remaining LMGC is provided [8]. All series on LM NNNC emphasize that liver resection in these patients should be seen as an integral part of cancer treatment (chemotherapy, radiotherapy and surgery) [2, $6,8]$. Chemotherapy is used on almost $100 \%$ of patients with LMGC during the pre- or post-operative period [5, $17,20]$. The main problem in evaluating these papers is that their main objective was to estimate the outcome of resection of LM NNNC globally, not just LMGC, so that information that can be obtained is low $[3,6-8,15]$.

Published series devoted exclusively to LMGC resection are scarce, and we can divide into those about all kinds of LMGC [1,5], and those dedicated to a specific tumor. Among the series on LMGC, Chi published a series of 12 patients in 1997 that included 7 LM OC, 2 from cervical cancer, 2 from endometrial cancer, and one from fallopian tube [5]. LM was unique in $92 \%$ of the cases. All LMGC were metachronous and were diagnosed with an average of 32 months disease-free period. Sixtyfour percent had resectable extrahepatic disease that was resected in combination with liver surgery. Seventy-five percent of hepatectomies performed were major liver resections. The morbidity was $8 \%$. The median survival obtained was 27 months [5]. Seventy-five percent of patients had recurrence of the disease, but only $22 \%$ were liver recurrences. Chi et al. were unable to obtain clear prognostic factors that indicated which patients would benefit from resection of LMGC. Unique LM, a prolonged disease-free period, and the absence of extrahepatic disease appear to be associated with a better pro- gnosis [5].

In the second group of articles devoted to a specific LM tumor, we highlight these series.

\section{LM from Ovarian Cancer}

The presence of an OC LM with no other single or multiple foci of disease is very uncommon [12,17]. In this situation, hepatic resection can potentially provide an improvement in survival time $[2,4,14,21]$. But LM usually occurs concomitantly with extrahepatic disease (peritoneal and/or other locations). This clinical situation is not considered an absolute contraindication for liver resection, but creates more reluctance to perform liver surgery $[5,22]$.

A percentage of patients with OC debut with advanced stages (III or IV) $[4,11,16,19,23]$, which are associated with a poor prognosis [16]. A combined treatment of primary debulking surgery and chemotherapy may be able to obtain a complete clinical response, but recurrence is common [4,12,14,21]. A second debulking surgery as treatment for tumor recurrence also obtains therapeutic benefits, but is not recommended if the disease-free period is less than 12 months [2,4,12,14,21,23]. It has also been shown that in patients without complete cytoreduction, but with residual disease less than $5 \mathrm{~cm}$ a survival of 30 months is achieved [24]. A high percentage of patients where the second cytoreduction is indicated, will have an LM, but in this second surgical procedure, liver resection is performed in only in $0 \%-9 \%$ of patients, demonstrating a clear reluctance for hepatectomy in patients with recurrent OC. There is no scientific evidence to show that the presence of LM implies a worse prognosis in patients with stage IV [2,4,12,21]. Moreover, patients with perihepatic and intraperitoneal implants in 
which an optimal debulking surgery (residual disease $<1$ $\mathrm{cm}$ ) could be performed, a better survival is obtained compared with those that cannot be surgically treated (survival 50 months vs 7 months) [10,11,16,23,25].

When a patient dies of OC disease progression, in 50\% of the cases metastatic liver disease is found at autopsy $[5,12,16,23,26]$. This suggests that liver resection should have a more important role as part of the treatment of patients with recurrent OC [16].

The OC can produce liver metastases in two ways. The most frequent and rapid spread is due to exfoliated cells that are implanted into the surface of the abdominal organs, which correspond to liver implants on the Glisson capsule $[4,10,11,17]$. The second track, more rare, is the blood stream, causing parenchymal LM, and is the main subject of this review $[4,11,17]$. The rate of this type of LM caused by the OC is lower than that caused by other malignancies, such as colon cancer [27]. The LM of epithelial OC is less frequent than OC LM stromal or germ cell LM [10]. In addition to the routes of spread are different, and the therapeutic approach is different, hence it is important to distinguish between parenchymal perihepatic metastases or implants on the liver surface with varying degrees of infiltration of the liver parenchyma [23,25].

Several histological variants of OC LM have been operated on: adenocarcinoma, papillary serous carcinoma, endometrioid carcinoma, clear cell carcinoma, granulosa cell mixed Mullerian tumor [4,5,11,21,23,27]. Any author could show survival after LM resection and the various histologic types, probably because the small sample size of the series [2].

The diagnosis of OC LM is often obtained using Computed Tomography (CT). The two more commons types of LM OC are well-defined cystic lesions or solid masses [10]. In the CT, it is important to distinguish between perihepatic implants, with or without infiltration of the liver parenchyma, and parenchymal LM [28]. Ninety percent of the implants did not show parenchymal infiltration, but it is important to know this information before surgery, as that patients with parenchymal infiltration require a liver resection [11,28]. The increase in CA125 is also useful in the diagnosis of LM [10,17] The role of Positron Emission Tomography (PET) is not fully defined, but probably PET-CT will be consolidated as an important test in the staging of patients with LM OC, especially to rule out metastatic extrahepatic foci.

The OC patients with LM have frequently received multiple lines of chemotherapy and several surgeries (primary debulking...) prior to considering liver resection $[21,23]$. The clinical scenarios are varied depending on whether the disease is synchronous or metachronous, if there is peritoneal disease that requires cytoreduction, or has already been done, according to the type of hepatectomy (minor or major), and the number and location of LM $[16,22,23]$.

The published series on metachronous LM OC (Table 2) consist of a small number of patients, between 8 and 26. The average age ranges between 51 to 62 years. The disease-free period prior to the hepatectomy was between 29 to 48 months. Sixty-five percent are single lesions. In $37.5 \%$ - 90\% hepatectomy was associated with resection of extrahepatic disease in other sites. The size is varied, measuring 1 to $18 \mathrm{~cm}$. The margin of liver resection was R0 in $50 \%$ to $100 \%$. Morbidity ranges from $0 \%$ to $21 \%$ with a mortality of $0 \%$. The recurrence rate was 50 to $80 \%$. Average actuarial survival was 26 - 62 months [2,4, $11,14,21]$.

Most of the authors suggest that liver resection, when extrahepatic disease is present, should be able to acquire a complete resection or at least optimal $(<1 \mathrm{~cm})$ of the liver and existing extrahepatic disease (4). In the published cases, some type of debulking surgery or resection of adjacent organs to the liver with hepatectomy was most often performed $[5,14]$.

The combination of major hepatectomy and debulking has been performed, but this increases the morbidity $[10,16,22]$. A hepatectomy in the cytoreduction interval is not accepted by all authors [16,23]. Patients with diffuse liver and aggressive involvement do not benefit from liver resection [4]. There is no indication on what to do in those with significant liver, but limited peritoneal,

Table 2. Liver metastasis from ovarian cancer series.

\begin{tabular}{ccccccccccccc}
\hline & $\mathrm{n}$ & Age & DFS & Unique LM & EHD & Size (cm) & Major LR & Morbidity & Mortality & R0 & Relapse & SV-months \\
\hline Merideth, 2003 [21] & 26 & 62 & 29 & & $77 \%$ & & $31 \%$ & $7.7 \%$ & $0 \%$ & & 26.3 \\
Yoon, 2003 [4] & 24 & 53 & 36,5 & $17 / 24$ & $75 \%$ & 5 & $16 \%$ & $21 \%$ & $0 \%$ & $54 \%{ }^{*}$ & $80 \%$ & - \\
Abood, 2008 [2] & 10 & 51 & 48 & - & $90 \%$ & 4,7 & $90 \%$ & $10 \%$ & $0 \%$ & $50 \%$ & - & 33 \\
Lim, 2009" [1] & 14 & 54 & - & - & & - & $50 \%$ & $0 \%$ & $0 \%$ & $43 \%{ }^{*}$ & $51 \% 5 y$ \\
Pekmekci, 2010 [14] & 8 & 56 & 39 & $8 / 8$ & $37.5 \%$ & 6 & - & $0 \%$ & $0 \%$ & $100 \%$ & $50 \%$ & 62 \\
\hline
\end{tabular}

DFS: disease free survival; LM: liver metastasis; EHD: extrahepatic disease; LR: liver resection; SV: survival; ${ }^{*}$ If peritoneal liver implants are included; ${ }^{* *}$ R0 including liver and other sites. 
disease [4].

Only a few prognostic factors have been found in the published series, and they vary between series $[2,4,14]$. Abood et al. observed that LM larger than $5 \mathrm{~cm}$ had a longer survival. They postulated a possible statistical bias. A negative resection margin in liver resection and absence of extrahepatic disease has also been postulated as important prognostic factor [2,21]. Loizzi et al., in their series of 29 patients treated with chemotherapy suggested that, mainly the tumor cell type, performance status, number of liver lesions, the presence of other sites besides the liver, and treatment with platinum-based chemotherapy were prognostic factors [17]. The patients that benefit from liver resection $[2,4,12,17,21]$ are those in good clinical condition and have a favorable tumor biology: stable disease, disease limited to the liver or extrahepatic resectable, slow-growing tumors, long disease-free period (>12 months) $[4,12]$. Neither the number of LM or patient age should be considered absolute contraindications for liver resection [12]. The absence of control groups in published series also do not lets us know the real benefit of surgery for LM of OC [4].

Radiofrequency ablation or cryoablation for treatment of a single LM from OC may be useful as an alternative to resection, although it has very rarely been used [12,23, 27]. Its use in combination with hepatectomy may allow the indications for surgery to be extended, treating more LM, especially in patients requiring major hepatectomy and cytoreductive surgery [23].

\section{Other LMGC Different from OC LM}

- CERVICAL CANCER: LM from cervical cancer are rare $(1.2 \%$ - 2.2\%) [12]. Kim et al., in a series of 1665 cervical carcinomas, found that only $1.2 \%$ developed LM, and only in 1 case was there an isolated LM without extrahepatic metastatic foci [29]. The LM autopsy rate is higher $15.8 \%-27.4 \%$ [12]. The average time of LM diagnosis is 39 months. The survival of untreated patients with LM is 10 months, and $0 \%$ at 2 years. Favorable prognostic factors are, the absence of extrahepatic disease, pelvic disease control, disease-free interval of more than 2 years, unilobar disease, and the use of chemotherapy [12]. Neither size nor the number of lesions influences the prognosis. The existence of extrahepatic disease is not an absolute contraindication for surgery [12]. The small number of patients is unable to define which patients actually benefit from resection of cervical cancer LM.

- ENDOMETRIAL CANCER: The number of resected LM published is very small. None of the conclusions obtained can be scientifically valid [12].

- OVARIAN TERATOMA: The rate of LM involvement in ovarian teratoma is $28 \%$ [30]. They are usu- ally metastatic deposits in the liver surface, although they can coexist with perihepatic LM and solid intrahepatic lesions. The resection is recommended if the size of perihepatic deposits is less than $15 \mathrm{~cm}$ [30].

- OVARIAN GRANULOSA CELL TUMORS: They have a low incidence $(5 \%-6 \%)$ of LM [26,31]. They are usually multifocal with extensive liver parenchyma involvement [31].

- UTERINE SARCOMA: In a series of 66 LM of sarcomas from different locations, 3 were located in the uterus [32]. In this series, it states that the LM from sarcoma are not usually responsive to chemotherapy or chemoembolization, so the surgery free margin should be considered the best therapeutic option [32].

\section{Conclusion}

True LMGC, not peritoneal implants in the liver, usually appear in patients with disseminated disease, therefore liver resection is uncommon. In some patients with only liver disease or resectable limited extrahepatic disease, liver resection is a safe alternative and improves the oncological results. It is necessary the decision should be tailored for each patient.

\section{REFERENCES}

[1] O. Kollmar, M. R. Moussavian, S. Richter, M. Bolli and M. K. Schilling, "Surgery of Liver Metastasis in Gynecological Cancer-Indication and Results,” Onkologie, Vol. 31, No. 7, 2008, pp. 375-379

[2] G. Abood, M. Bowen, R. Potkul, G. Aranh and M. Shoup, "Hepatic Resection for Recurrent Metastasic Ovarian Cancer,” American Journal of Surgery, Vol. 195, No. 3, 2008, pp. 370-373. doi:10.1016/j.amjsurg.2007.12.012

[3] S. A. Earle, E. A. Perez, J. C. Gutierrez, D. Sleeman, A. S. Livingstone, D. Franceschi, et al., "Hepatectomy Enables Prolonged Survival in Select Patients with Isolated Noncolorectal Liver Metastasis," Journal of the American College of Surgeons, Vol. 203, No. 4, 2006, pp. 436-446. doi:10.1016/j.jamcollsurg.2006.06.031

[4] S. S. Yoon, W. R. Jarnagin, Y. Fong, R. P. DeMatteo, R. R. Barakat, L. H. Blumgart and D. S. Chi, "Resection of Recurrent Ovarian or Fallopian Tuve Carcinoma Involving the Liver,” Gynecologic Oncology, Vol. 91, No. 2, 2003, pp. 383-388. doi:10.1016/j.ygyno.2003.07.005

[5] D. S. Chi, Y. Fong, E. S. Venkatraman and R. R. Barakat, "Hepatic Resection for Metastasic Gynecologic Carcinomas," Gynecologic Oncology, Vol. 66, No. 1, 1997, pp. 4551. doi:10.1006/gyno.1997.4727

[6] S. K. Reddy, A. S. Barbas, C. Marroquin, M. A. Morse, P. C. Kuo and B. M. Clary, "Resection of Non Colorectal Nonneuroendorine Liver Metastases: A Comparative Analysis," Journal of the American College of Surgeons, Vol. 204, No. 3, 2007, pp. 372-382. doi:10.1016/j.jamcollsurg.2006.12.019

[7] T. R. O’Rourke, P. Tekkis, S. Yeung, J. Fawcett, S. Lyn- 
ch, R. Strong, et al., "Long-Term Results of Liver Resection for Non-Colorectal, Non-Neuroendocrine Metastases,” Annals of Surgical Oncology, Vol. 15, No. 1, 2008, pp. 207-218. doi:10.1245/s10434-007-9649-4

[8] R. Adam, L. Chiche, T. Aloia, D. Elias, R. Salmon, M. Rivoire, et al., "Hepatic Resection for Noncolorectal Nonendocrine Liver Metastases,” Annals of Surgery, Vol. 244, 2006, pp. 524-535.

[9] M. Schmelzle, C. F. Eisenberger, S. Schulte, H. Matthaei, M. Krausch and W. T. Knoefel, "Non-Colorectal, NonNeuroendocrine, and Non-Sarcoma Metastases of the Liver: Resection as a Promising Tool in the Palliative Management," Langenbeck's Archives of Surgery, Vol. 395, No. 3, 2010, pp. 227-234. doi:10.1007/s00423-009-0580-y

[10] N. Kawagishi, Y. Shirihata, K. Ishida, K. Satoh, Y. Enomoto, Y. Akamatsu, et al., "Hepatic Resection of Giant Metastatic Tumor from Clear Carcinoma of the Ovary,” Journal of Hepato-Biliary-Pancreatic Surgery, Vol. 12, No. 2, 2005, pp. 155-158. doi:10.1007/s00534-004-0957-9

[11] M. C. Lim, S. Kang, K. S. Lee, S. S. Han, S. J. Park, S. S. Seo and S. Y. Park, "The Clinical Significance of Hepatic Parenchymal Metastasis in Patients with Primary Epitelial Ovarian Cancer,” Gynecologic Oncology, Vol. 112, No. 1, 2009, pp. 28-34. doi:10.1016/j.ygyno.2008.09.046

[12] S. Tangjitgamol, C. F. Levenback, U. Beller and J. J. Kavanagh, "Role of Surgical Resection for Lung, Liver, and Central Nervous System Metastases in Patients with Gynecological Cancer: A Literature Review,” International Journal of Gynecological Cancer, Vol. 14, No. 3, 2004, pp. 399-422. doi:10.1111/j.1048-891x.2004.14326.x

[13] D. R. Carpizo and M. D’Angelica, "Liver Resection for Metastasic Colorectal Cancer in the Presence of Extrahepatic Disease," Annals of Surgical Oncology, Vol. 16, No. 9, 2009, pp. 2411-2421. doi:10.1245/s10434-009-0493-6

[14] S. Pekmezci, K. Saribeyoglu, E. Aytac, M. Arvas, F. Demirkiran and M. Ozguroglu, "Surgery for Isolated Liver Metastasis of Ovarian Cancer," Asian Journal of Surgery, Vol. 33, No. 2, 2010, pp. 83-88. doi:10.1016/S1015-9584(10)60014-0

[15] G. Ercolani, G. L. Grazi, M. Ravaioli, G. Ramacciato, M. Cescon, et al., "The Role of liver Resections for Noncolorectal Nonneuroendocrine Metastases: Experience with 142 Observed Cases,” Annals of Surgical Oncology, Vol. 12, No. 6, 2005, pp. 1-8. doi:10.1245/ASO.2005.06.034

[16] D. S. Chi, S. M. Temkin, N. R. Abu-Rustum, P. Sabattini, W. Jarnagin and L. H. Blumgart, "Major Hepatectomy at Interval Debulking for Satge IV Ovarian Carcinoma: A Case Report,” Gynecologic Oncology, Vol. 87, No. 1, 2002, pp. 138-142. doi:10.1006/gyno.2002.6717

[17] V. Loizzi, C. Rossi, G. Cormio, A. Cazzolla, D. Altomare and L. Selvaggi, "Clinical Features of Hepatic Metastasis in Patients with Ovarian Cancer," International Journal of Gynecological Cancer, Vol. 15, No. 1, 2005, pp. 2631. doi:10.1111/j.1048-891x.2005.14406.x

[18] National Comprehensive Cancer Network. http://www.nccn.org/professionals/physician_gls/pdf/ovar ian.pdf

[19] National Comprehensive Cancer Network. http://www.nccn.org/professionals/physician_gls/pdf/uter ine.pdf

[20] E. Vibert and E. Castaing, "Surgical Treatment of Liver Metastases of Gynecological Cancer: Local Treatment of a Systemic Disease,” Onkologie, Vol. 31, No. 8-9, 2008, pp. 425-426. doi:10.1159/000143274

[21] M. A. Merideth, W. A. Cliby, G. L. Keeney, T. G. lesnick, D. M. Nagorney and K. C. Podratz, "Hepatic Resection for Metachronous Metastases from Ovarian Carcinoma," Gynecologic Oncology, Vol. 89, No. 1, 2003, pp. 16-21. doi:10.1016/S0090-8258(03)00004-0

[22] G. E. Chalkiadakis, K. G. Lasithiotakis, I. Petrakis, C. Kourousis and V. Georgoulias, "Major Hepatectomy and Right Hemicolectomy at Thetime of Primary Cytoreductive Surgery for Advanced Ovarian Cancer: Report of a Case,” International Journal of Gynecological Cancer, Vol. 15, No. 6, 2005, pp. 1115-1119. doi:10.1111/j.1525-1438.2005.00169.x

[23] R. Mateo, G. Singh, N. Jabbour, S. Palmer, Y. Genyk and L. Roman, "Optimal Cytoreduction after Combined Resection and Radiofrequency Ablation of Hepatic Metastases from Recurrent Malignant Ovarian Tumors," Gynecologic Oncology, Vol. 97, No. 1, 2005, pp. 266-270. doi:10.1016/j.ygyno.2004.12.038

[24] W. E. Winter, L. Maxwell, C. Tian, M. J. Sundborg, G. S. Rose, P. G. Rose, et al., "Tumor Residual after Surgical Cytoreduction in Prediction of Clinical Outcome in Stage IV Epithelial Ovarian Cancer: A Gynecological Oncology Group Study,” Journal of Clinical Oncology, Vol. 26, No. 1, 2008, pp. 83-89. doi:10.1200/JCO.2007.13.1953

[25] R. E. Bristow, F. J. Montz, L. D. Lagasse, R. S. Leuchter and B. Y. Karlan, "Survival Impact of Cytoreduction in Stage IV Epithelian Ovarian Cancer,” Gynecologic Oncology, Vol. 72, No. 3, 1999, pp. 278-287.

doi:10.1006/gyno.1998.5145

[26] P. G. Rose, M. S. Piver, Y. Tsukada and T. S. Lau, "Metastastic Patterns in Histologic Variants of Ovarian Cancer. An Autopsy Study,” Cancer, Vol. 64, No. 7, 1989, pp. 1508-1513. doi:10.1002/1097-0142(19891001)64:7<1508::AID-CNC R2820640725>3.0.CO;2-V

[27] I. A. Jacobs, C. K. Chang and G. Salt, "Hepatic Radiofrequency Ablation of Metastatic Ovarian Granulosa Cell Tumors,” American Journal of Surgery, Vol. 69, No. 5, 2003, pp. 416-418.

[28] O. Akin, E. Sala, C. S. Moskowitz, N. Ishill, R. A. Soslow, D. S. Chi and H. Hricak, "Perihepatic Metastases from Ovarian Cancer: Sensitivity and Specificity of CT for the Detection of Metastases with and Those without liver Parenchymal Invasion,” Radiology, Vol. 248, 2008, pp. 511-517. doi:10.1148/radiol.2482070371

[29] G. E. Kim, S. W. Lee and C. O. Suh, "Hepatic Metastases from Carcinoma of the Uterine Cervix," Gynecologic Oncology, Vol. 70, No. 1, 1998, pp. 56-60. doi:10.1006/gyno.1998.5037

[30] Q. Fan, H. Huang, L. Lian and J. Lang, “Characteristics, 
Diagnosis, and Treatment of Hepatic Metastasis Pure Inmature Ovarian Teratoma," Chinese Medical Journal, Vol. 114, No. 5, 2001, pp. 506-509.

[31] T. K. Madhuri, S. Butler-Manuel, N. Karanjia and A. Tailor, "Liver Resection for Metastases Arising from Recurrent Granulosa Cell Tumor of the Ovary-A Case Series,” European Journal of Gynaecological Oncology, Vol.
31, No. 3, 2010, pp. 342-344.

[32] T. Pawlik, T. Vauthey, E. Abdalla, R. Pollock, L. M. Ellis and S. A. Curley, "Results of a Single Center Experience with Resection and Ablation for Sarcoma Metastatic to the Liver,” Archives of Surgery, Vol. 141, No. 6, 2006, pp. 537-544. 